\title{
Damage indices evaluation for one-dimensional guided wave-based structural health monitoring
}

\author{
Lucas S. Barreto $^{\text {a }}$ (D), Marcela R. Machado ${ }^{a *}$ (D), Juliana C. Santos a ${ }^{(D)}$, Braion B. de Moura ${ }^{\text {a }}$ (D), Leila Khalij ${ }^{\text {b }}$ (D) \\ a Departamento de Engenharia Mecânica, Universidade de Brasilia, 70910-900 Brasilia, Brasil. E-mail: lucas.sv.barreto@gmail.com, \\ marcelam@unb.br, julianasaaantos@gmail.com, braionbarbosa@gmail.com

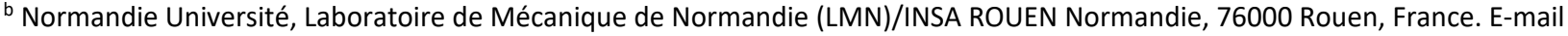 \\ leila.khalij@insa-rouen.fr \\ * Corresponding author
}

https://doi.org/10.1590/1679-78256292

\begin{abstract}
Damage detection in structures and systems is essential for monitoring parameters that can affect their integrity. This paper evaluates the efficiency of four damage indices (DIs) commonly used with temporal wave signals. The root mean square deviation, mean absolute percentage deviation, covariance, and correlation coefficient deviation DIs are presented, and a normalization is then proposed. An Euler-Bernoulli beam is used as a guided wave modelled with the spectral element method and excited by a toneburst signal. It includes the theoretical background of the throw-off beam, undamaged and cracked beam spectral elements. The DIs for a single crack position and a map varying crack depth and positions are calculated with deterministic and random temporal signal responses derived from noise addition. Results showed that DIs could identify and quantify the damage conditioned to the pulse location point and the influence of noise in the estimation, which leads in an analysis comparable to practical applications.
\end{abstract}

\section{Keywords}

Damage quantification, Spectral Element Method, Wave propagation, Damage Index.

\section{Graphical Abstract}
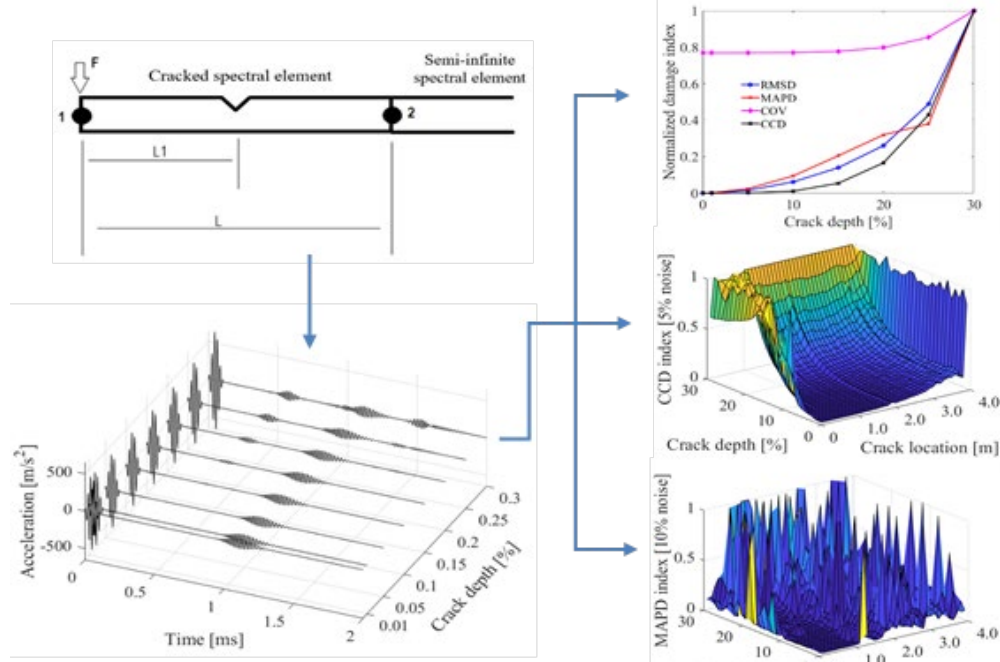

Received October 15, 2020. In revised form January 16, 2021. Accepted February 01, 2021. Available online February 05, 2021 https://doi.org/10.1590/1679-78256292

(a) Latin American Journal of Solids and Structures. ISSN 1679-7825. Copyright @ 2021. This is an Open Access article distributed under the terms of the Creative Commons Attribution License, which permits unrestricted use, distribution, and reproduction in any medium, provided the original work is properly cited. 


\section{INTRODUCTION}

Damage detection and structure health monitoring (SHM) are an extensive subject in the literature. The motivation to detect and monitor damages at the earliest stage helps the prognosis and decision process regarding the maintenance of the systems (Balageas et al., 2010). Non-destructive techniques are widely used and directly affect typical issues regarding the design of new structures, the repair and monitoring of existing ones. This approach in health monitoring has large acceptance in the engineering communities. Mainly because the SHM techniques provide orientation on structural descriptions or material behaviour for the mathematical models, regarding this feature, with repeated tests over time, the methods indicate the appearance of discontinuities (damage) occurring during the structure's lifetime.

Many non-destructive damage detection methods have been developed and improved over the years and these are meant to monitor and evaluate the structural health in different engineering sectors. These methods render a more effective time-based maintenance (Sohn et al., 2003; Gopalakrishnan et al., 2011). Among the damage detection techniques, the elastic-wave-based considers the wave scattering phenomena and mode conversion caused by damage to monitor the structure. Therefore, the quantitative evolution of damage can be achieved by examining the scattered wave signals. This approach is cost-effective, fast, repeatable, sensitive to small damage, and it can detect surface and internal damage. It can also perform the mechanical waves propagation over significant distances with high sensitivity to discontinuities near the wave path. In their book, Su and Ye (2009) presented a comprehensive description of damage identification techniques using Lamb waves from fundamental theory to engineering applications. Mitra and Gopalakrishnan (2016), and Ostachowicz et al. (2012) addressed a review of the theory, applications of damage modelling, and guided wave propagation in SHM. Giurgiutiu (2014) published a complete book with theory and application about SHM methods using piezoelectric wafer active sensors (PWAS). The author provided the readers with a comprehensive overview of piezoelectric material and sensors, vibration analysis, elastic and guided waves, applications of the PWAS, signal processing, and pattern recognition.

The spectral element method (SEM) was formulated from the analytical solution of the wave displacement equation written in the frequency domain (Doyle, 1997; Lee, 2009). Due to the SEM being the exact solution of differential equations governing the problem, it became a suitable technique to model structural damage detection. In general, changes in either global or local structural properties can be associated with an imperfection or damage. This behaviour can be explained by the presence of a crack in the structure introducing local flexibility that affects its response. It also generates more evident changes in the elastic waves that propagate in the structure. Elastic wave propagation in a damaged structure at medium and high-frequency bands using SEM was presented by Krawczuk et al. (2002), Krawczuk (2002), Palacz and Krawczuk (2002), Ostachowicz (2008), Machado et al. (2019), Dutkiewicz and Machado (2019), and Zhang et al. (2018). Palacz (2018) published an overview of spectral methods to model and use wave propagation in damage detection problems. Ng et al. (2011), Flynn et al. (2012), Machado and Santos (2015), Machado et al. (2017), and Machado et al. (2018) proposed a structural damage detection including random parameters and stochastic models.

Damage is the change made to a system that adversely affects its current or future performance (Farrar and Worden, 2006). This concept of damage involves a comparison between two different states of a system. Several approaches have been developed to define and extract signal characteristics in other areas in turn identify structural damage, and among these techniques, damage indices are highlighted. Damage indices or damage index (DI), are the most straightforward damage detection algorithm that consists of comparing two entire signals and returning a scalar value, which indicates a level of damage present in the structure. The development of Dls and damage identification algorithms remains an open area for the practical application of structural monitoring (Ho and Ewins, 2000; Giurgiutiu, 2014). Su and Ye (2009) enumerated characteristics of signals from Lamb waves that can be used in the development of DIs including propagation time, root mean square value (Rizzo and Lanza, 2006; Ghaffarzadeh et al., 2016), signal variance (Rizzo and Lanza, 2006), peak-to-peak amplitude (Su and Ye, 2009), strain energy attenuation rate (Sharma et al., 2006; Apetre et al., 2008), local impedance (Lee and Staszewski, 2003), among others. The comparison of the DIs method to perform damage detection has complications related to scale and amplitudes or the inexistence of magnitude, which renders it difficult to evaluate. Numerous Dls were proposed by using the wave propagation in timedomain. The root mean square deviation (RMSD) or also called Euclidean norm (Giurgiutiu and Rogers, 1998; Park et al., 2006; Su and Ye, 2009; DeLuca et al., 2020), the mean absolute percentage deviation (MAPD) (Tseng and Naidu, 2002), covariance (COV) (Monaco et al., 2000; Tseng and Naidu, 2002), and the correlation coefficient deviation (CCD) (Tung and Yen, 2005; Giurgiutiu, 2014; Stawiarski and Muc, 2019) are the most used DIs.

In this paper, the SEM is used to model an Euler-Bernoulli beam, with and without damage, connected to a throwoff beam spectral element. It therefore aims to analyse and compare the damage indices RMSD, MAPD, COV and CCD related to the crack location, depth, and over the influence of noise. For reliable comparison over the DIs, according to efficiency and accuracy, we proposed the normalised DIs, which vary between zero and a unity, where zero means no 
damage. The temporal wave propagation signals obtained from the numerical model are used to calculate the DIs and perform the damage prognosis. Aside from the punctual crack position and Dls estimation, we presented a DI map analysis covering crack position over the beam length and depth of cracks varying from 0 to $30 \%$ of damage. The map provides a detailed behaviour of each DI under parametric variations combined with noise.

\section{DAMAGE INDICES}

The DIs are formulated by comparing a reference signal, usually derived from the system considered undamaged or with a healthy signature, to the one provided by the system under the presence of discontinuing or damage. In this aspect, various approaches have been developed to define and extract signal features in different domains to identify structural damage based on an indicator that describes the damage. The DIs are associated with the estimation techniques for damage quantification and reveals important information about the structural health condition. The most common feature extraction used for response-signal processing falls into the second category, which is the damage assessment. The variance and the RMS are notable features for statistical analysis, signal magnitude, or energy estimation. In structural monitoring, they are also used as a DI. The DIs based on the RMS value, $D I_{R M S}$, and on the variance, $D I_{\text {Variance, }}$, are defined as (Su and Ye, 2009)

$D I_{R M S}=\sqrt{\frac{\sum_{i=1}^{N} x_{i}^{2}}{N}}$

$D I_{\text {Variance }}=\frac{1}{N-1} \sum_{i=1}^{N}\left(x_{i}-\bar{x}\right)^{2}$,

where $\bar{x}$ represents the medium magnitude of the discrete wave time signal with $N$ sample points and $x_{i}(i=1,2, \ldots N)$ are the elements of the spectrum. $D I_{R M S}$ is a damage index associated with the signal energy, while $D I_{V a r i a n c e}$ indicates its variability compared to the average magnitude value. However, the $D I_{R M S}$ and $D I_{V a r i a n c e}$ indices only evaluate one signal at a time, which does not allow a comparison between intact and damaged states of the structure. The RMSD is the combination of both (Su and Ye, 2009) and it can perform a comparison of two signals described as

$R M S D=\sqrt{\frac{\sum_{i=1}^{N}\left(y_{i}-x_{i}\right)^{2}}{\sum_{i=1}^{N} x_{i}^{2}}}$

where $y_{i}(i=1,2, \ldots N)$ is the response captured under another state of the structure, for instance, when damage is present. The RMSD index is the most appropriate damage index to observe two states of a structure because it includes the signal energy combined with the variance between them (Tseng and Naidu, 2002). Another DI called MAPD also correlates two signals $\left(x_{i}\right.$ and $\left.y_{i}\right)$ and evaluates the average deviations at each point of the response defined by (Tseng and Naidu, 2002)

$M A P D=\frac{1}{N} \sum_{i=1}^{N}\left|\frac{y_{i}-x_{i}}{x_{i}}\right|$,

The covariance evaluates the weighted product of the deviations from their respective average. It determines the relationship between two signals by multiplying corresponding elements and returning a scalar. Mathematically, the covariance is estimated as (Giurgiutiu, 2014)

$\operatorname{COV}=\frac{1}{N-1} \sum_{i=1}^{N}\left(y_{i}-\bar{y}\right)\left(x_{i}-\bar{x}\right)$,

by correlating the response signals of the undamaged and damaged structure, a damage metric can be characterised based on the deviation between the signature. In this case, the covariance is a DI representing the relationship between the compared spectra (Giurgiutiu, 2014). An issue that should be checked is the signal time lag to secure similar time instant. Therefore, pre-signal processing may be considered before application of the DIs. There are many techniques to deal with this problem, e.g. the envelope of the signal (Su and Ye, 2009; Al-Oudatallah et al., 2017), signal denoising via discrete wavelet transform (Giurgiutiu, 2014) or similar signal processing procedure. The DI related to the CCD measures 
the linear relationship between the two signals that are similar to the COV. The difference is that the CCD is normalised to be independent of the unit of measurement (Tung and Yen, 2005). It can be described as (Giurgiutiu, 2014)

$C C D=1-\frac{\sum_{i=1}^{N}\left(y_{i}-\bar{y}\right)\left(x_{i}-\bar{x}\right)}{\sqrt{\sum_{i=1}^{N}\left(y_{i}-\bar{y}\right)^{2} \sum_{i=1}^{N}\left(x_{i}-\bar{x}\right)^{2}}}$,

The DIs are used to detect the damage as well as its growth. Because each DI has a unique scale, a direct comparison among them would not be accurate. This paper treated the normalised DIs by the maximum value of the indexes related to the crack depth. The values vary between zero to unity, when zero means no damage and up to unity indicates the presence of cracks or anomalies. Therefore, a vector containing the RMSD values estimated for different crack depth $(\alpha)$ is collected in a vector and normalised by the max value, as expressed in

$\boldsymbol{R M S D}=\left[R M S D_{\alpha 1}, R M S D_{\alpha 2}, \ldots, R M S D_{\alpha n}\right]$

$\boldsymbol{R} \boldsymbol{M S} \boldsymbol{D}_{\text {normalised }}=\frac{1}{\max \{\boldsymbol{R M S D}\}} \boldsymbol{R M S D}$.

where $R M S D_{\alpha n}$ is a scalar number calculated with an specific crack death $(\alpha n=1,2, \ldots, n)$. A similar procedure was applied for the MAPD, where $\boldsymbol{M A P D}=\left[M A P D_{\alpha 1}, M A P D_{\alpha 2}, \ldots, M A P D_{\alpha n}\right]$ is the vector containing the DI estimated for $\alpha i$ cracks depth. The normalised $\boldsymbol{M} \boldsymbol{A P} \boldsymbol{D}_{\text {normalised }}$ will be

$\boldsymbol{M A P D}_{\text {normalised }}=\frac{1}{\max \{\boldsymbol{M A P D}\}} \boldsymbol{M A P D}$.

Likewise, $\operatorname{COV}=\left[\operatorname{COV}_{\alpha 1}, \operatorname{COV}_{\alpha 2}, \ldots, \operatorname{COV}_{\alpha n}\right]$ is a vector containing the DI of each crack depth calculated with Equation (5). For normalised $\mathbf{C O V}$ it has

$\operatorname{COV}_{\text {normalised }}=\frac{1}{\max \{\mathrm{COV}\}} \operatorname{COV}$.

and the normalised $\boldsymbol{C C} \boldsymbol{D}_{\text {normalised }}$ is obtained from the vector $\boldsymbol{C C D}=\left[C C D_{\alpha 1}, C C D_{\alpha 2}, \ldots, C C D_{\alpha n}\right]$ divided by its maximum value as

$\boldsymbol{C C} \boldsymbol{D}_{\text {normalised }}=\frac{1}{\max \{C \boldsymbol{C D}\}} \boldsymbol{C C D}$.

\section{SPECTRAL ELEMENT METHOD}

In dynamic system analysis and SHM, it is crucial to have an efficient and economical numerical technique. Finite Element Method (FEM) is one of the most common computational methods employed in several scientific areas. However, in medium and high-frequency wave propagation problems, this method demands very high computational costs. Narayanan and Beskos (1978) first proposed the SEM, further improved and named SEM by Doyle (1997) and Lee (2009). SEM is a frequency-domain formulation, and the element interpolation function is the exact solution of the wave equation. This characteristic and the spectral domain make SEM more suitable to solve the crack problem. The advantage of SEM is the reduced number of elements required to model the system when compared to other computational methods, as demonstrated in Figure 1.

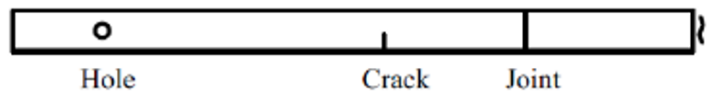

(a)

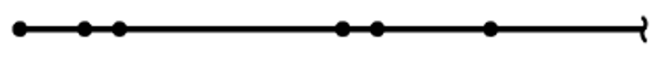

(b)

Figure 1 Representation: (a) Physical structure; (b) Spectral element model. 
SEM is similar in style to the FEM written in the frequency domain, and the element interpolation function is the exact analytical solution of the differential equation, which implies a high accuracy of the system approximation. Based on this latter aspect, the number of elements required for a spectral model will coincide with the number of structural discontinuities. Aside from the low computation cost, the effectiveness in dealing with frequency-domain issues in the non-reflecting boundary conditions of the infinite or semi-infinite-domain problems are advantages of this method (Lee, 2009). Nonetheless, the exact wave solutions are not available for certain types of structure. In this case, the application of SEM becomes infeasible and other techniques, such as the Wave Finite Element method (WFEM) or FEM, are necessary.

\subsection{Beam spectral element}

The beam illustrated in Figure 2 is assumed to be slender with transversal and rotational nodal displacement, shear and momentum nodal forces. By neglecting shear deformations, the differential equation of movement in its spectral form is written as (Doyle,1997)

$\frac{d^{4} \hat{v}}{d x^{4}}-\beta^{4} \hat{v}=0$

with the homogeneous solution given by

$\hat{v}(x)=\mathrm{a}_{1} e^{-i k x}+\mathrm{a}_{2} e^{-k x}+\mathrm{a}_{3} e^{-i k(L-x)}+\mathrm{a}_{4} e^{-k(L-x)}=\mathbf{e}(x, \omega) \mathbf{a}$,

where

$\mathbf{e}(x, \omega)=\left[e^{-i k x}, e^{-k x}, e^{-i k(L-x)}, e^{-k(L-x)}\right]$,

$\mathbf{a}=\left[\mathrm{a}_{1}, \mathrm{a}_{2}, \mathrm{a}_{3}, \mathrm{a}_{4}\right]^{T}$

where $\mathbf{a}$ is the vector of arbitrary constants that will be determined to satisfy the associated boundary conditions (Lee, 2009), $L$ is the beam length and $k$ the wavenumbers given by

$\beta^{2} \equiv \sqrt{\frac{\omega^{2} \rho S}{E I}}, \quad k= \pm \beta$ or $\pm i \beta$,

where $\omega=(2 \pi \mathrm{f})$ is the circular frequency, $\mathrm{f}$ is the ordinary frequency, $E$ is Young's modulus, $S$ is the cross-section area, $\rho$ is the density, $I$ is the moment of inertia, and $i=\sqrt{-1}$. By using a complex Young's modulus, $E_{c}=E(1+i \eta)$, internal structural damping is introduced where $\eta$ is the hysteretic structural loss factor. Figure 2 illustrates a two-node healthy beam spectral element model with two degrees of freedom (dof) per node. The nodal displacements and forces are represented by $\hat{v}_{1}, \hat{v}_{2}, \widehat{\phi}_{1}, \widehat{\phi}_{2}$, and $\widehat{V}_{1}, \widehat{V}_{2}, \widehat{M}_{1}, \widehat{M}_{2}$, respectively.

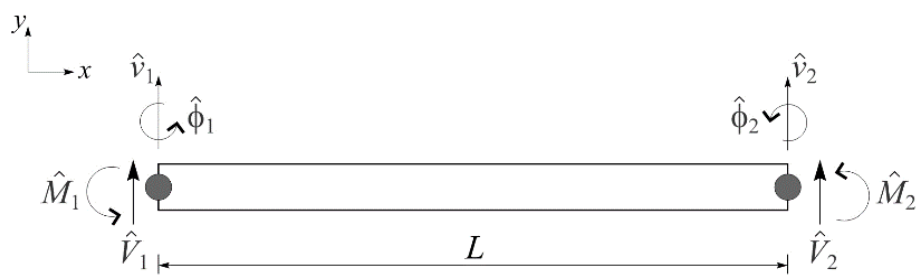

Figure 2 Two nodes beam spectral element.

The spectral nodal displacements of the beam related with the displacement field as at node $1(x=0)$ and at node $2(x=L)$ gives 
$\boldsymbol{d}=\left\{\begin{array}{l}\hat{v}_{1} \\ \hat{\phi}_{1} \\ \hat{v}_{2} \\ \hat{\phi}_{2}\end{array}\right\}=\left\{\begin{array}{c}\hat{v}(0) \\ \hat{v}^{\prime}(0) \\ \hat{v}(L) \\ \hat{v}^{\prime}(L)\end{array}\right\}=\left\{\begin{array}{c}\mathbf{e}(0, \omega) \\ \mathbf{e}^{\prime}(0, \omega) \\ \mathbf{e}(L, \omega) \\ \mathbf{e}^{\prime}(L, \omega)\end{array}\right\} \mathbf{a}=\boldsymbol{H}_{\boldsymbol{B}}(\omega) \mathbf{a}$,

where $\mathbf{a}=\boldsymbol{H}_{\boldsymbol{B}}(\omega)^{-1} \boldsymbol{d}$, and

$\boldsymbol{H}_{\boldsymbol{B}}(\omega)=\left[\begin{array}{cccc}1 & 1 & e^{-i k L} & e^{-k L} \\ -i k & -k & i k e^{-i k L} & k e^{-k L} \\ e^{-i k L} & e^{-k L} & 1 & 1 \\ -i k e^{-i k L} & -k e^{-k L} & i k & k\end{array}\right]$.

The frequency-dependent displacement within an element is interpolated from the nodal displacement vector $\boldsymbol{d}$, by eliminating the constant vector $\mathbf{a}$ for the relation $\mathbf{a}=\boldsymbol{H}_{\boldsymbol{B}}(\omega)^{-1} \boldsymbol{d}$, and it is expressed as

$\hat{v}(\mathrm{x})=\mathbf{g}(x, \omega) \boldsymbol{d}=\mathbf{e}(x, \omega) \boldsymbol{H}_{B}^{-1}(\omega) \boldsymbol{d}$.

where the interpolation function is $\mathbf{g}(x, \omega)=\mathbf{e}(x, \omega) \boldsymbol{H}_{B}^{-1}(\omega)$. Shear forces and bending moments defined for the beam are related to the defined forces and moments in a spectral nodal form as

$\boldsymbol{f}=\left\{\begin{array}{c}\widehat{V}_{1} \\ \widehat{M}_{1} \\ \widehat{V}_{2} \\ \widehat{M}_{2}\end{array}\right\}=\left\{\begin{array}{c}-\widehat{V}(0) \\ -\widehat{M}(0) \\ \widehat{V}(L) \\ \widehat{M}(L)\end{array}\right\}=\left\{\begin{array}{c}-E I \hat{v}^{\prime \prime \prime}(0) \\ -E I \hat{v}^{\prime \prime}(0) \\ E I \hat{v}^{\prime \prime \prime}(L) \\ E I \hat{v}^{\prime \prime}(L)\end{array}\right\}=\left\{\begin{array}{c}\mathbf{e}^{\prime \prime \prime}(0, \omega) \\ \mathbf{e}^{\prime \prime}(0, \omega) \\ \mathbf{e}^{\prime \prime \prime}(L, \omega) \\ \mathbf{e}^{\prime \prime}(L, \omega)\end{array}\right\} \mathbf{a}$,

whereby applying boundary conditions provides

$\boldsymbol{f}(\omega)=E I\left[\begin{array}{cccc}-i k^{3} & k^{3} & i e^{-i k L} k^{3} & -e^{-k L} k^{3} \\ -k^{2} & k^{2} & -e^{-i k L} k^{2} & -e^{-k L} k^{2} \\ i e^{-i k L} k^{3} & -e^{-k L} k^{3} & -i k^{3} & k^{3} \\ -e^{-i k L} k^{2} & e^{-k L} k^{2} & -k^{2} & k^{2}\end{array}\right] \mathbf{a}=\boldsymbol{G}(\omega) \mathbf{a}$.

By replacing the $\mathbf{a}=\boldsymbol{H}_{\boldsymbol{B}}(\omega)^{-1} \boldsymbol{d}$ in Equation (14), we relate the nodal forces to the nodal displacement

$\boldsymbol{f}=\boldsymbol{G}(\omega) \boldsymbol{H}_{B}^{-1}(\omega) \boldsymbol{d}=\boldsymbol{S}_{B}(\omega) \boldsymbol{d}$,

where $\mathbf{S}_{\mathrm{B}}(\omega)=\mathbf{G}(\omega) \mathbf{H}_{\mathrm{B}}^{-1}(\omega)$ is the dynamic stiffness matrix of the Euler-Bernoulli beam spectral element.

\subsection{Semi-infinite or throw-off beam spectral element}

The semi-infinite or throw-off beam spectral element is a particular case to an intact beam where node 2 tends to infinity as represented in Figure 3.

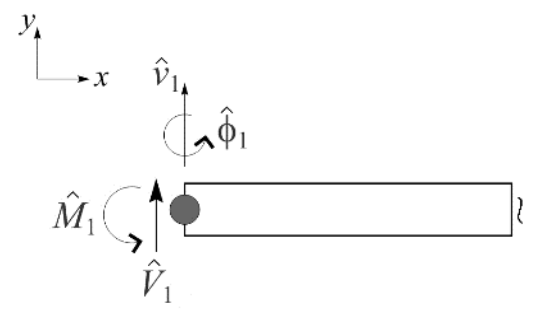

Figure 3 Throw-off beam spectral element. 
The theory of the throw-off beam spectral element is similar to the beam spectral element theory when the spectral dynamic stiffness matrix is (Doyle,1997)

$\boldsymbol{S}_{t}(\omega)=\frac{E I}{L^{3}}\left[\begin{array}{cc}(i-1) k^{3} & i k^{2} \\ i k^{2} & (i-1) k^{3}\end{array}\right]$.

\subsection{Cracked beam spectral element}

The beam spectral element with a transversal and non-propagating crack (Krawczuk, 2002) is illustrated in Figure 4. The element contains two nodes with two degrees of freedom per node, where $L$ is the beam length, $L_{1}$ is the crack position from node 1 , and ' $a$ ' is the crack depth related to the cross-section high. The crack is modelled by dimensionless local flexibility represented by $\theta$, which is calculated by Castigliano's theorem and the laws of fracture mechanics.

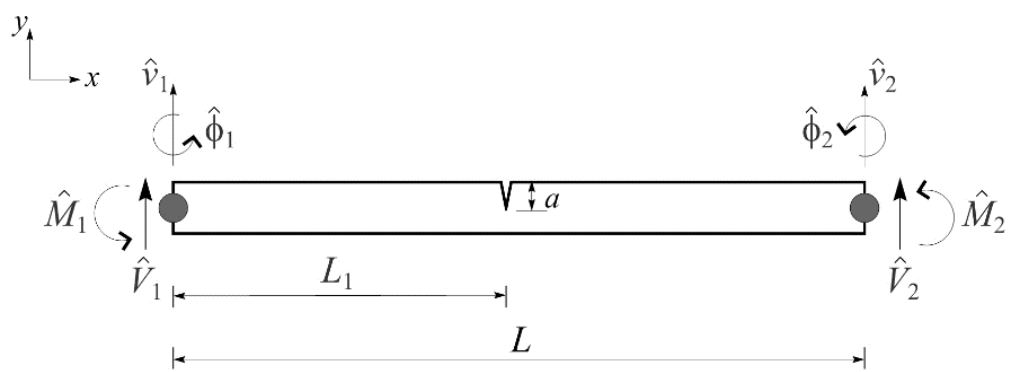

Figure 4 Cracked beam spectral element.

The solution applied to this element is divided into two parts, the left $\hat{v}^{l}(x)$ and the right $\hat{v}^{r}(x)$ side crack. Therefore, the nodal displacements are

$\hat{v}^{l}(x)=\mathrm{a}_{1} e^{-i(k x)}+\mathrm{a}_{2} e^{-(k x)}+\mathrm{a}_{3} e^{-i k\left(L_{1}-x\right)}+\mathrm{a}_{4} e^{-k\left(L_{1}-x\right)}\left[0 \leq x \leq L_{1}\right]$,

$\hat{v}^{r}(x)=\mathrm{a}_{5} e^{-i k\left(L_{1}+x\right)}+\mathrm{a}_{6} e^{-k\left(L_{1}+x\right)}+\mathrm{a}_{7} e^{-i k\left(L-\left(L_{1}+x\right)\right)}+\mathrm{a}_{8} e^{-k\left(L-\left(L_{1}+x\right)\right)}\left[0 \leq x \leq L-L_{1}\right]$.

The coefficients $\mathrm{a}_{i}$, $(i=1: 8)$ can be calculated as a function of the nodal spectral displacements, considering the boundary conditions for the element. Thus, for the cracked beam element, the boundary conditions at the ends of the beam and the crack position must be considered as follows

- $\quad$ On the left side of the element for $x=0$ :

$\hat{v}^{l}(x)=\hat{v}_{1} \quad \frac{\partial \hat{v}^{l}(x)}{\partial x}=\hat{\phi}_{1}$

- $\quad$ In the crack position, displacement and rotation are considered for $\hat{v}^{l}(x)$. So, $x=L_{1}$ and for $\hat{v}^{r}(x)$ where $x=0$ :

$\hat{v}^{l}(x)=\hat{v}^{r}(x)$

$\frac{\partial \hat{v}^{r}(x)}{\partial x}-\frac{\partial \hat{v}^{l}(x)}{\partial x}=\theta \frac{\partial^{2} \hat{v}^{r}(x)}{\partial x^{2}}$,

$\frac{\partial^{2} \hat{v}^{l}(x)}{\partial x^{2}}=\frac{\partial^{2} \hat{v}^{r}(x)}{\partial x^{2}}$

$\frac{\partial^{3} \hat{v}^{l}(x)}{\partial x^{3}}=\frac{\partial^{3} \hat{v}^{r}(x)}{\partial x^{3}}$

- $\quad$ On the right side of the element for $x=L-L_{1}$ : 
$\hat{v}^{r}(x)=\hat{v}_{2} \quad \frac{\partial \hat{v}^{r}(x)}{\partial x}=\hat{\phi}_{2}$

Then, considering the equations that describe the nodal spectral displacements, Equations (19) to (21), to the left and right of the crack, applying the boundary conditions and writing in matrix form

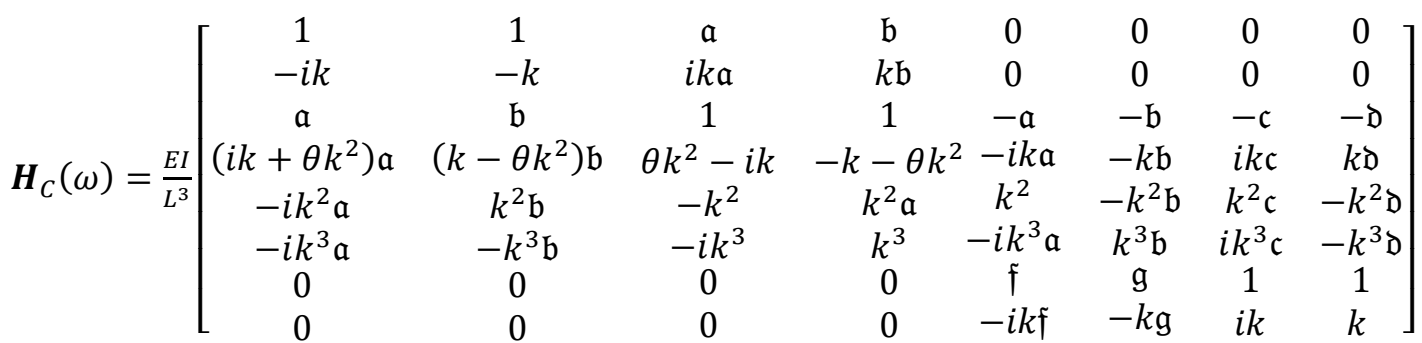

where $\mathfrak{a}=e^{i k L_{1}}, \mathfrak{b}=e^{-k L_{1}}, \mathfrak{c}=e^{-i k\left(L-L_{1}\right)}, \mathfrak{d}=e^{-k\left(L-L_{1}\right)}, \mathfrak{f}=e^{-i k L}, \mathfrak{g}=e^{-k L}$.

Equation (26) falls into the general formulation of the element, where $\boldsymbol{d}=\boldsymbol{H}_{C}(\omega) \mathbf{a}$. The coefficients can be related to nodal spectral displacements by

$\mathrm{a}_{i}=H_{c i 1}^{-1} \hat{v}_{1}+H_{c i 2}^{-1} \hat{\phi}_{1}+H_{c i 3}^{-1} \hat{v}_{2}+H_{c i 4}^{-1} \hat{\phi}_{2}$

At this point, the $\boldsymbol{H}_{C}(\omega)$ matrix, originally of $8 \times 8$ dimension, is reduced to an $8 \times 4$ matrix. This process occurs according to Equation (27), where $H_{c i j}^{-1}$ represents the elements of the inverse matrix of Equation (26). The nodal spectral forces can be represented by differentiating the spectral displacements $\hat{v}^{l}$ and $\hat{v}^{r}$ related to $x$, and, in this case, generally represented by $\boldsymbol{f}=\boldsymbol{G}(\omega)$ a. In the matrix form, we have

$$
\left\{\begin{array}{l}
\hat{f}_{1} \\
\hat{f}_{2} \\
\hat{f}_{3} \\
\hat{f}_{4}
\end{array}\right\}=\left[\begin{array}{cccccccc}
i k^{3} & -k^{3} & -i k^{3} \mathfrak{a} & k^{3} & 0 & 0 & 0 & 0 \\
-k^{2} & k^{2} & -k^{2} \mathfrak{a} & k^{2} & 0 & 0 & 0 & 0 \\
0 & 0 & 0 & 0 & i k^{3} \mathfrak{f} & -k^{3} \mathfrak{g} & -i k^{3} & k^{3} \\
0 & 0 & 0 & 0-k^{2} \mathfrak{f} & k^{2} \mathfrak{g} & -k^{2} & k^{2}
\end{array}\right]\left\{\begin{array}{l}
\mathrm{a}_{1} \\
\mathrm{a}_{2} \\
\mathrm{a}_{3} \\
\mathrm{a}_{4} \\
\mathrm{a}_{5} \\
\mathrm{a}_{6} \\
\mathrm{a}_{7} \\
\mathrm{a}_{8}
\end{array}\right\}
$$

From the nodal forces and displacements, Equations (28) and (26), the frequency-dependent dynamic stiffness matrix of the Euler-Bernoulli beam element with an open, non-propagating crack related to the nodal forces and displacements is,

$\boldsymbol{f}_{[4 \times 1]}=\boldsymbol{G}_{c}(\omega)_{[4 \times 8]} \boldsymbol{H}_{c}^{-1}(\omega)_{[8 \times 4]} \boldsymbol{d}=\boldsymbol{S}_{\boldsymbol{c}}(\omega)_{[4 \times 4]} \boldsymbol{d}$,

where $\mathbf{S}_{\mathbf{c}}(\omega)$ is a $4 \times 4$ cracked beam dynamic stiffness matrix.

\subsection{Local crack flexibility}

The crack flexibility coefficient $\theta$ is calculated using Castigliano's theorem, where the flexibility in the crack position for a one-dimensional beam spectral element can be obtained by (Tada et al., 1973)

$c=\frac{\partial^{2} U}{\partial P^{2}}$

where $U$ denotes the elastic deformation energy due to cracking, and $P$ is the element's nodal load. Considering that only crack mode $I$ is present in the beam element, the elastic deformation energy can be expressed as (Tada et al., 1973)

$U=\frac{1-v^{2}}{E} \int_{S_{c}} K_{I}^{2} d S_{c}$ 
which $v$ is the Poisson's ratio, $S_{c}$ is the cracked area, and $K_{I}$ is a stress intensity factor corresponding to mode $I$ of the crack, which can be represented by (Tada et al., 1973)

$K_{I}=\frac{6 M}{b h^{2}} \sqrt{\pi \alpha} f\left(\frac{\alpha}{h}\right)$

where $b$ is the base and $h$ the height of the beam cross-section, $\alpha$ is the variation in the crack depth, as shown Figure 5 , $M$ is the bending moment in crack position, and $\mathrm{f}$ is a correction function of the mode I stress intensification factor, which can be written as (Tada et al., 1973)

$f\left(\frac{\alpha}{h}\right)=\sqrt{\frac{2 h}{\pi \alpha} \tan \left(\frac{\pi \alpha}{2 h}\right)} \frac{0.923+0.199\left(1-\sin \left(\frac{\pi \alpha}{2 h}\right)\right)^{4}}{\cos \left(\frac{\pi \alpha}{2 h}\right)}$.
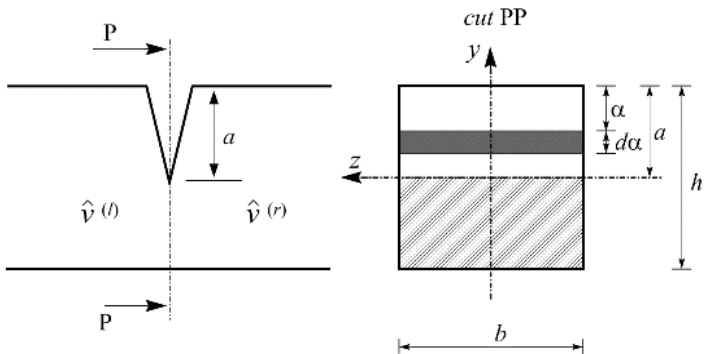

Figure 5 Cross-section of the cracked beam in the crack position.

The coefficient $\mathrm{c}$ that calculates the crack flexibility is expressed as follows (Tada et al., 1973)

$c=\frac{72 \pi}{b h^{2}} \int_{0}^{\alpha} \alpha f^{2}(\alpha) d \alpha$

where $\alpha=\frac{a}{h}$. Finally, the dimensional local flexibility is given by

$\theta=\frac{E I C}{L}$

\section{NUMERICAL RESULTS}

The simulations were performed in the MATLAB software and considered a steel beam with a length of $L=4 \mathrm{~m}$, a cross-section with a height of $h=0.03 \mathrm{~m}$, and width of $b=0.01 \mathrm{~m}$. The material properties are Young's modulus of $E=210 \mathrm{GPa}$, the material density of $\rho=7800 \mathrm{~kg} / \mathrm{m}^{3}$, and damping factor as $\eta=0.01$. The structural damping was modelled by a complex elastic modulus, $E=E(1+i \eta)$. The numerical investigations verified the beam's structural integrity. The damages were simulated considering a non-propagating open crack. The beam has a free-free boundary condition connected to a throw-off spectral element, with a toneburst excitation pulse at the node 1 . The cracked and healthy beams, both with the same geometries and properties, are shown in Figure 6 (a-b).

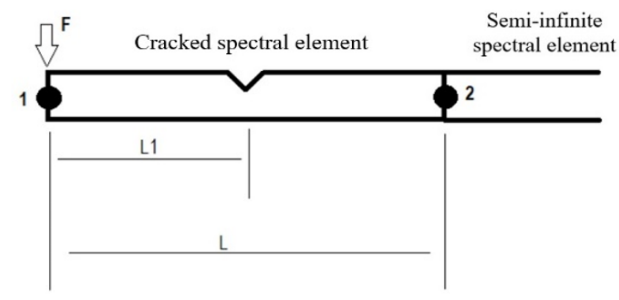

(a)

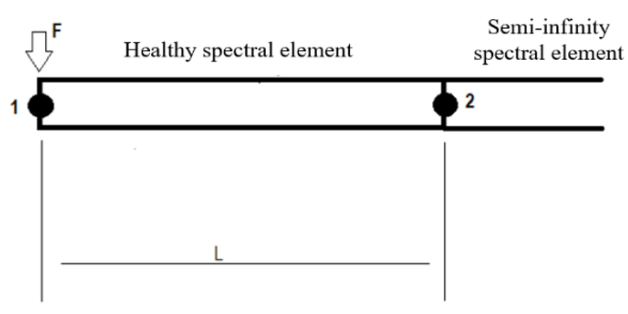

(b)

Figure 6: Structural representation: (a) Cracked beam models; (b) Healthy beam models. 
The impulsive pulse force improves the visualisation of the wave propagating through the beam and indicates a possible discontinuity or crack. The excitation toneburst signal was obtained with a sine function of 20 periods modulated by a Hanning window of $0.10 \mathrm{~ms}$ duration and a carrier frequency $\left(f_{c}\right)$ of $50 \mathrm{kHz}$. Figure 7 (a-b) shows the toneburst pulse signal in the time and frequency domains.

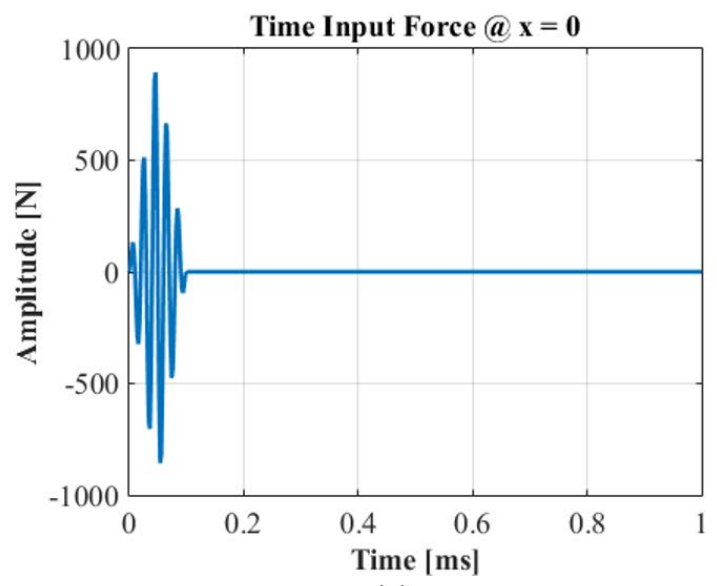

(a)

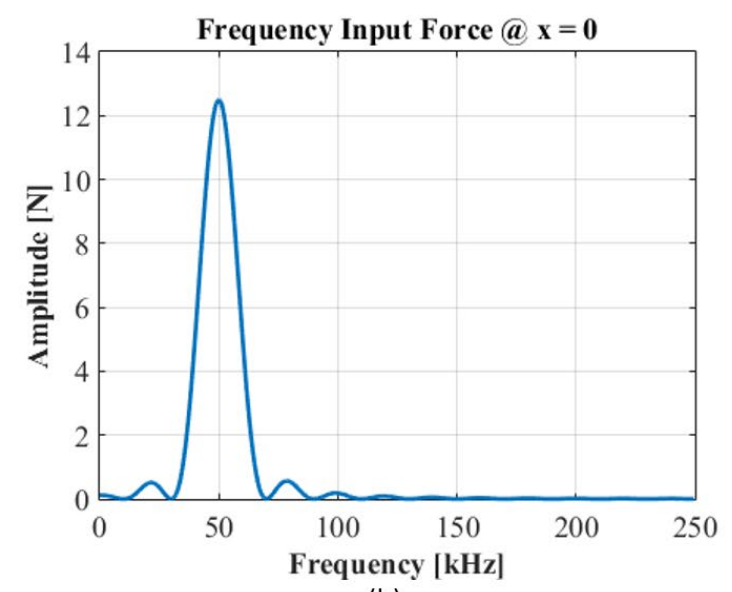

(b)

Figure 7: Excitation toneburst pulse $\left(f_{c}=50 \mathrm{kHz}\right)$ : (a) Excitation signal in the time domain; (b) Excitation signal in the frequency domain.

Excitation and response are applied at node 1 (see Figure 6). The wave travels through the structure until it reaches node 2 where it is reflected and continues through the throw-off element that dissipates the remaining energy of the system. The amplitude of vertical acceleration decreases throughout the structure's length due to internal damping $\eta$. Figure 8 (a) shows the temporal acceleration response for the healthy structure, which presents two pulses: one in the excitation time (node 1) of the incident wave, and the other in the reflection time (node 2). The temporal acceleration response of the cracked beam has two additional pulses, as shown in Figure 8 (b): one between the incident and reflection time and the other after the reflection time. These additional pulses characterise the waves that are partially reflected and transmitted due to a localised material discontinuity, which could indicate the existence of a discontinuity or crack. These results show that the SEM model can reproduce the wave propagation behaviour throughout the beam and therefore identify the crack.

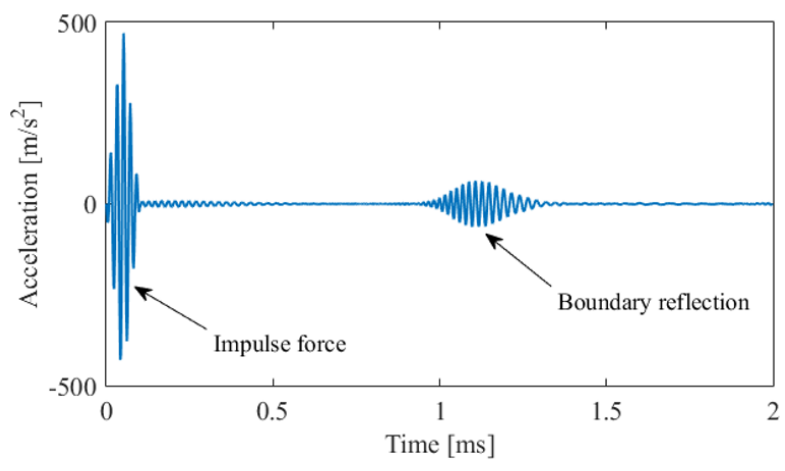

(a)

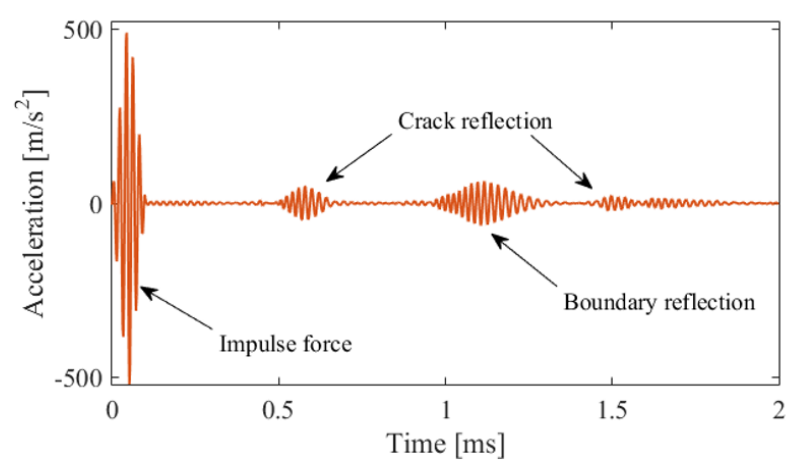

(b)

Figure 8: Acceleration response for (a) healthy beam; (b) Cracked beam with damage of $30 \%$ at $L_{1}=0.5 \mathrm{~L}$.

The influence of crack depth associated with the crack position in the cracked beam response is compared to the healthy beam. Seven values assumed for the crack depth range between $0 \%$ (no-crack), 1\%, 5\%, 10\%, 15\%, 20\%, 25\%, and $30 \%$ of the beam's cross-section height, and crack positions were chosen at $L_{1}=0.18 L, L_{1}=0.5 L$, and $L_{1}=0.82 L$ from node 1. Figure $9(\mathrm{a}-\mathrm{c})$ shows the temporal response estimated for each crack location and different crack depth. The curves obtained for each crack are displayed in parallel for better visualisation of the results. 


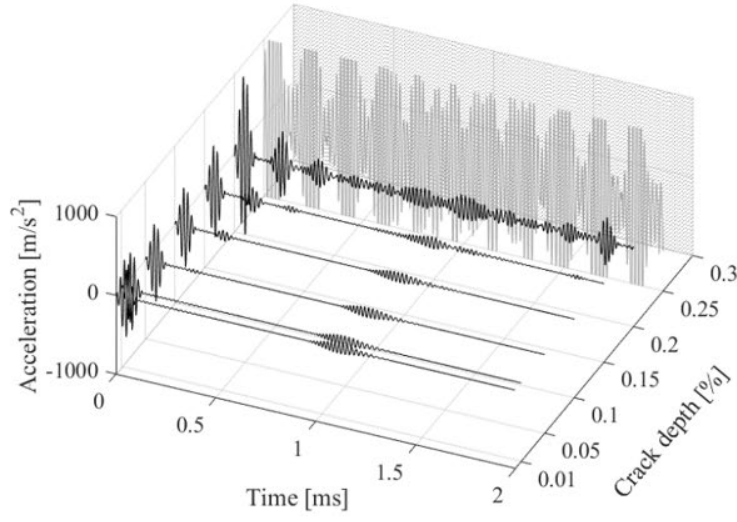

(a)

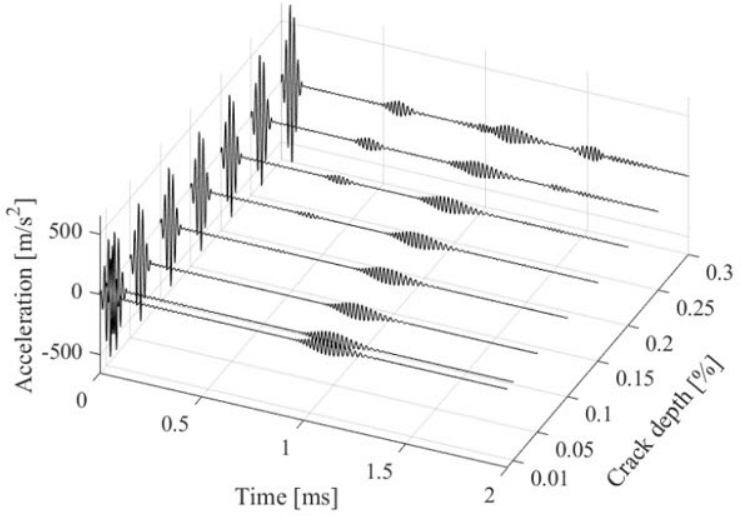

(b)

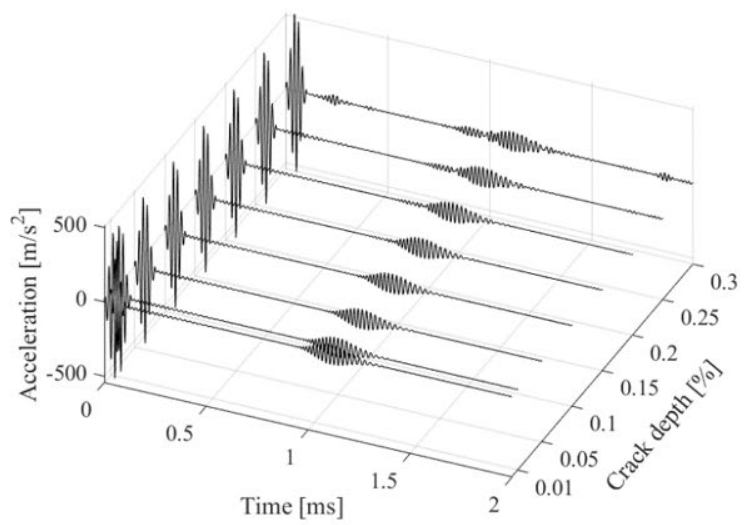

(c)

Figure 9: Acceleration temporal response for healthy and cracked beams with crack depth of $0 \%$ (no-crack), 1\%, 5\%, 10\%, 15\%, $20 \%, 25 \%$ and $30 \%$ at crack positions: (a) $L_{1}=0.18 \mathrm{~L}$, (b) $L_{1}=0.5 \mathrm{~L}$, and (c) $L_{1}=0.82 \mathrm{~L}$.

Figure 9 (a) shows the beam time response with the crack location at $L_{1}=0.18 \mathrm{~L}$ and crack depth varying from 0 to $30 \%$ of the cross-section height. In this case, the crack is located close to the excitation point. An additional pulse in the response with a crack of up to $5 \%$ is not identified, for a crack depth varying from $10 \%$ to $20 \%$ a pulse between the excitation and reflection time and after the reflection time is observed. It increases its amplitude as the crack grows. For a crack depth of $25 \%$ and $30 \%$, an instability over the time response leads to a numerical inconsistency and no physical meaning representation. In these cases, the time responses plotted in grey (dashed lines) increased in amplitude and it is therefore not possible to identify the impulse force, boundary, and crack reflections.

In Figure $9(\mathrm{~b})$, the crack position was set at $L_{1}=0.5 \mathrm{~L}$ and the crack depth varied from $0 \%$ to $30 \%$. The time response shows a clear reflection due to the crack in all cases. The crack expressing $10 \%$ of the beam's cross-section height exhibited one pulse between the excitation and boundary reflection, related to the crack, and another after the boundary reflection. The size of the crack increased the pulse amplitudes of the reflection. By locating the crack at $L_{1}=0.82 L$, the reflection because of the crack is closed to the wave reflection from the boundary, and the amplitude increased as the crack grew in depth. To achieve a more real analysis and to include possible randomness in the numerical model, presented in the acquisition of signals, we have included a white noise with $5 \%$ and $10 \%$ to the temporal responses.

A common practice in damage detection is to compare the response-signal of the undamaged structure with that of the damaged structure. Various techniques have been developed to define and extract signal features to identify structural damages. The DIs function as an indicator to describe the damage detection. The Dls that use time domain response derived from wave propagation are analysed in this section, where their advantages and limitations are explored. The RMSD, MAPD, COV, and CCD indices, applied for such analysis, presented differences in the amplitude scale or the inexistence of magnitude, rendering them difficult to be used and evaluated. We performed normalisation of the DIs by dividing the result to the highest absolute value found in each $\mathrm{DI}$, as demonstrated in Section 2. Therefore, the DIs is presented in values between zero to a unity, where zero accuses no damage and a higher value up to unity indicates the presence of a crack and its severity within the analysis scenario. 


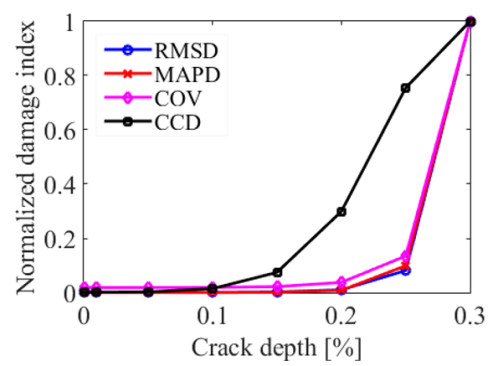

(a)

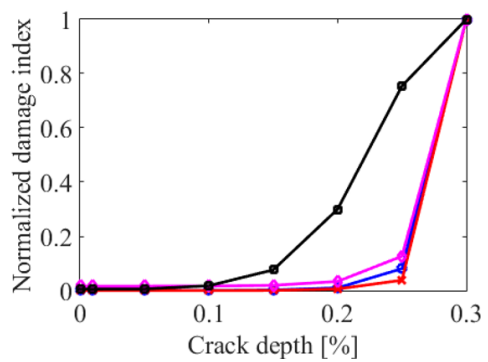

(b)

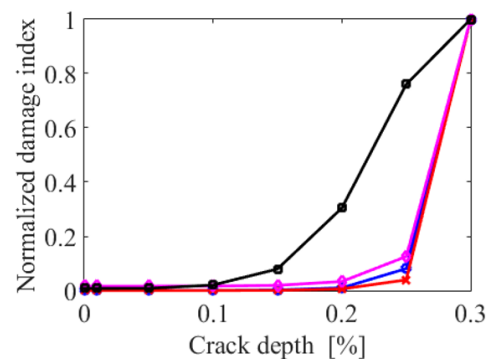

(c)

Figure 10: DIs comparison with the crack position at $L_{1}=0.18 \mathrm{~L}$, and crack depth ranging between $0 \%$ (no-crack), $1 \%, 5 \%, 10 \%$, $15 \%, 20 \%, 25 \%$, and $30 \%$ of the cross-section height. Responses without noise (a), $5 \%$ of noise (b), and $10 \%$ of noise (c).

The results of the DIs obtained for the crack locate it at $L_{1}=0.18 \mathrm{~L}$ and the crack depth ranging between $0 \%$ (no-crack), $1 \%, 5 \%, 10 \%, 15 \%, 20 \%, 25 \%$, and $30 \%$ of the beam's cross-section height are shown in Figure 10 (a-c). Therefore, the identification and quantification are not straightforward because the crack is located close to the excitation point (node 1). Figure $10(\mathrm{a})$ is the DIs comparison estimated with the responses without adding noise, Figure 10 (b) is the Dls estimated using the signals with $5 \%$ of noise and Figure 10 (c) with $10 \%$ of noise. With the crack close to excitation point and over $25 \%$ of the crack depth, the signal no longer corresponds to the studied phenomenon, as demonstrated in Figure 9(a). In all but the CCD, the damage indices were not sensitive to the crack depth. Moreover, the noise caused a small influence in the DIs results when the crack was located close to the excitation point because the impulse force has a high amplitude of the signal became dominant over DIs estimation, as demonstrated in the DI maps. The crack detection and severity are observed from $10 \%$ of crack depth. From this point on, it is impossible to track the crack growth analysing the DIs.

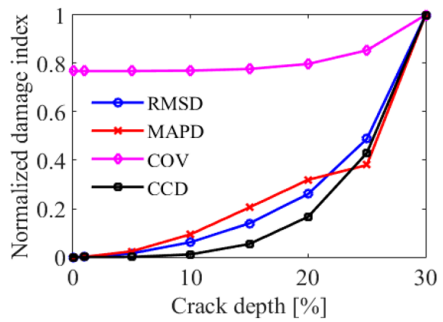

(a)

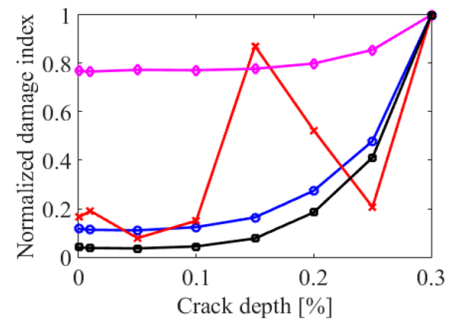

(b)

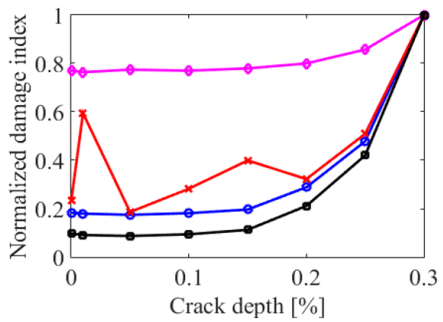

(c)

Figure 11: DIs comparison with the crack position at $L_{1}=0.5 \mathrm{~L}$, and crack depth ranging between $0 \%$ (no-crack), $1 \%, 5 \%, 10 \%, 15 \%$, $20 \%, 25 \%$, and $30 \%$ of the cross-section height. Responses without noise (a), $5 \%$ of noise (b), and $10 \%$ of noise (c).

Positioning the crack at $L_{1}=0.5 \mathrm{~L}$, with a reflection around $0.5 \mathrm{~ms}$, indicates the presence of the crack, as shown in Figure 11 (a-c). It is possible to clearly identify the crack at any depth over $15 \%$ and a crack depth under $10 \%$ may compromise the damage detection by visual inspection. For the signal without noise, the DIs increased exponentially as the crack depth grew, which fulfils the damage detection in each crack assessment. By adding 5\% and $10 \%$ of noise in the signals, RMSD, COV and CCD indices increased in the index amplitude caused by the noise and, with up to $15 \%$ of crack, there is no expressive changes in the DI level. MAPD index presented an abrupt change for the crack depth of $15 \%$ when it considered $5 \%$ of noise and an unstable estimation with $10 \%$ of noise. The COV damage index shows an unexpected index level. Even a small crack (as 1\%) reports a damage index of $77 \%$, with limited sensitivity to the increase in the crack depth. This instability is better seen in the DI map.

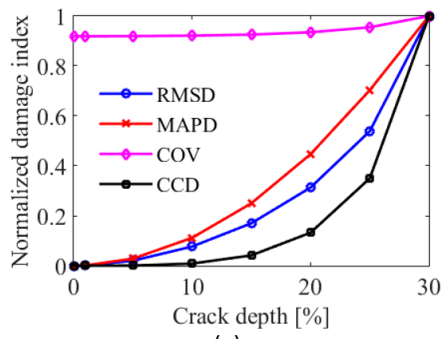

(a)

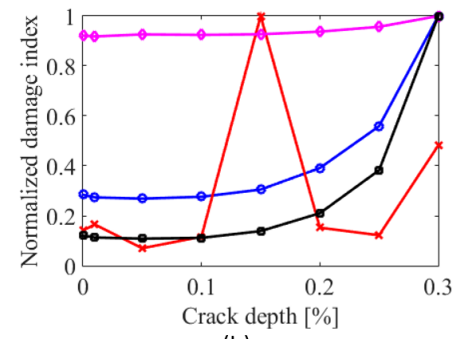

(b)

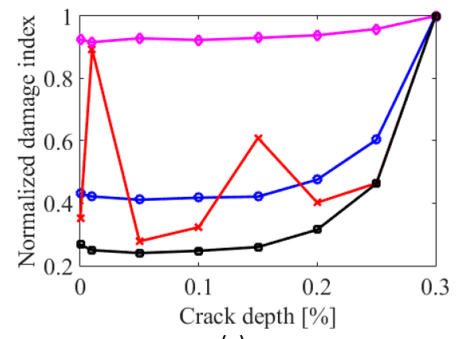

(c)

Figure 12: DIs comparison with the crack position at $L_{1}=0.82 \mathrm{~L}$, and crack depth ranging between $0 \%$ (no-crack), $1 \%, 5 \%, 10 \%$, $15 \%, 20 \%, 25 \%$, and $30 \%$ of the cross-section height. Responses without noise (a), $5 \%$ of noise (b), and $10 \%$ of noise (c). 
Figure 12 (a-c) shows the normalised DIs for the crack placed at $L_{1}=0.82 L$, further from the application force. The results followed the crack positioned in the middle of the beam. The RMSD and CCD indices were considered the most appropriate and accurate to assess the damage detection in the cases studied. Their values vary little as the crack location changes, and they are sensitive to the increase in crack depth. The MAPD index, disregarding the presence of noise, proves to be effective in the detection. However, by adding noise of $5 \%$ and $10 \%$, it showed inconsistency and failed to consider noticeable patterns that may indicate a correlation between the DI and the crack size. Finally, the COV index was ineffective in locating cracks over the range of $L_{1}=0.5 \mathrm{~L}$ and $L_{1}=L$. The index cannot be considered reliable to assess the damage severity for a beam with this configuration.

The tests were carried out for different crack depths and three different positions in Section 4. With the increase of the crack depth, the reflected wave became more evident in all cases. By relating the crack depth and location, it was possible to produce a DI map which improves the analysis. Therefore, graphs of the map in two and three dimensions cover the damage detection and better demonstrate the DI behaviour. The steps in both cases vary one-by-one over the entire length of the beam and up to $30 \%$ of depth. The time responses used to calculate the Dls were without noise, with $5 \%$ of noise, and $10 \%$ of noise.

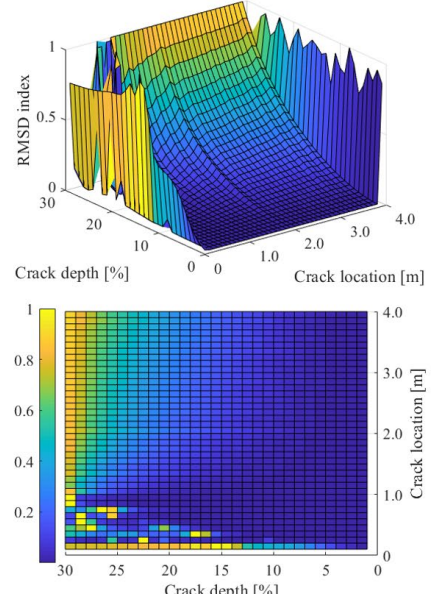

(a)
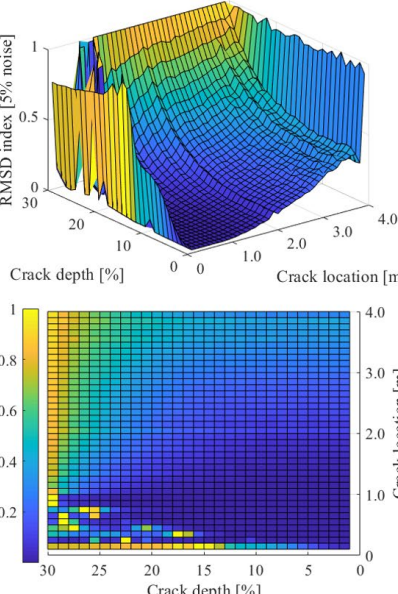

(b)

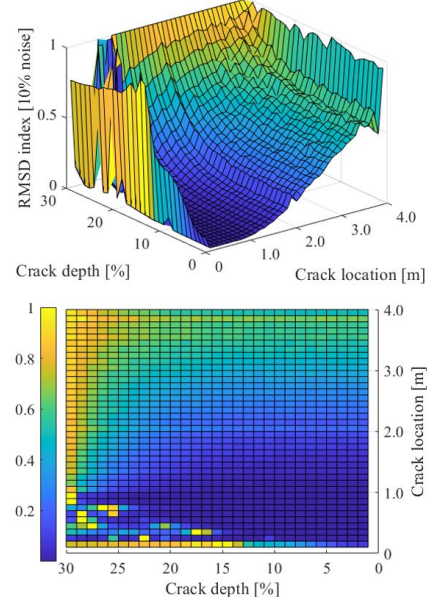

(c)

Figure 13: Two and three-dimension RMSD index map without noise (a), $5 \%$ of noise (b), and $10 \%$ of noise (c).

Damage indices map presented relevant results not seen in the single analysis. RMSD index maps in two and three-dimension, shown in Figure $13(\mathrm{a}-\mathrm{c})$, detected the damage over $5 \%$ in an area after $0.5 \mathrm{~L}$ and had an exponential surface following the crack growth, which shows the high sensitivity of this index. The addition of noise in the simulations barely influenced the behaviour of the RMSD index surfaces. It reduced the smoothness of the curve and increased the number of critical points without affecting the detection. Indeed, the noise significantly impacted the prognosis and must be considered in the entire monitoring process.
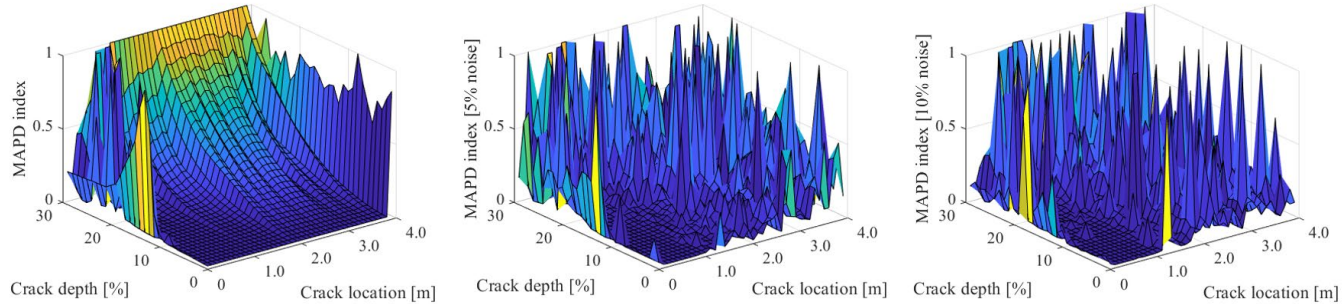

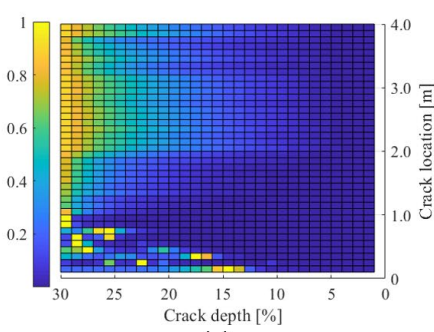

(a)

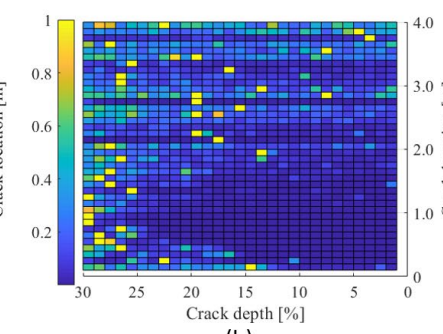

(b)

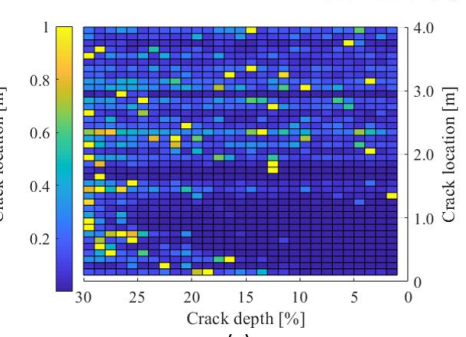

(c)

Figure 14: Two and three-dimension MAPD index map without noise (a), $5 \%$ of noise (b), and 10\% of noise (c).

The MAPD index maps presented in Figure $14(\mathrm{a}-\mathrm{c})$ were those with the most compromised performance by the noise. The MAPD estimated without noise presented an exponential surface following the crack growth, as expected, 
and presented sensitivity from the small to big crack depth. However, the DI estimation using the signal contaminated by the noise was inconsistent. This can be explained by the fact that the calculation was made considering the absolute variations between the two signals estimated from the healthy and damaged beams.
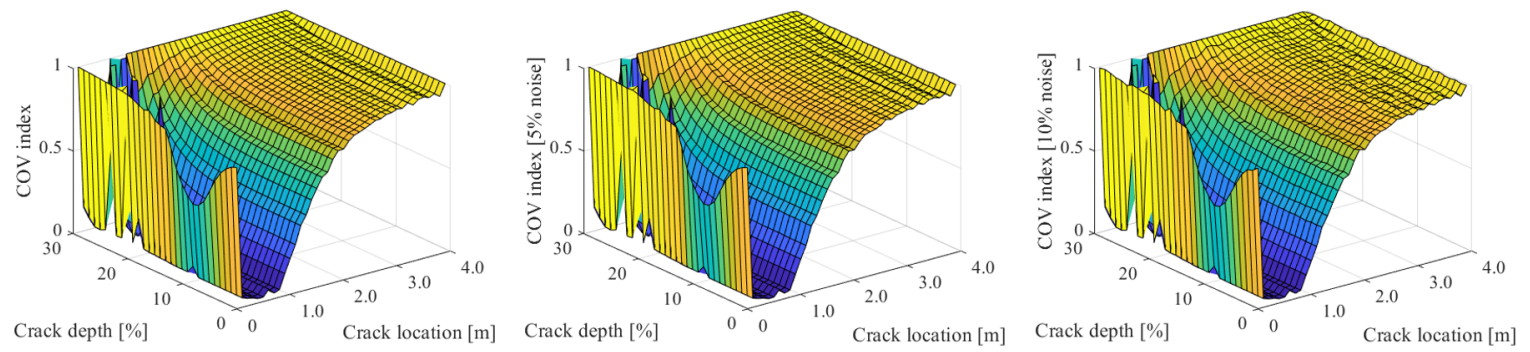

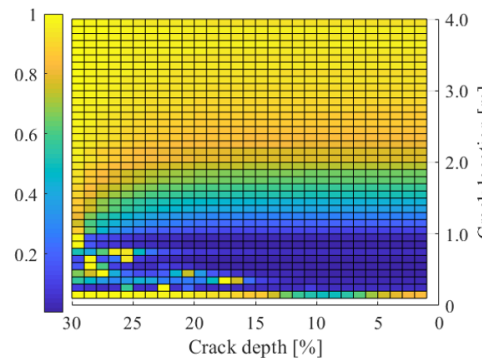

(a)

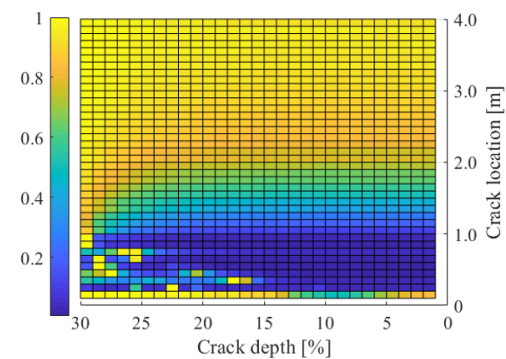

(b)

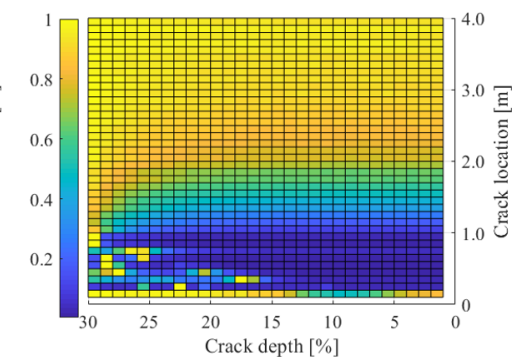

(c)

Figure 15: Two and three-dimension COV index map without noise (a), $5 \%$ of noise (b), and $10 \%$ of noise (c).

Figure 15 (a-c) shows the COV index maps, which had its DI values increasing with the crack depth, combined with the position, demonstrating less sensitivity to damage than the other DIs. Constant levels were maintained when the damage was located up to 1 meter in length, from one to two meters the index values increased even for small cracks, and over this region, it indicated a high index level. There were slight changes in the COV-DIs with the addition of noise.
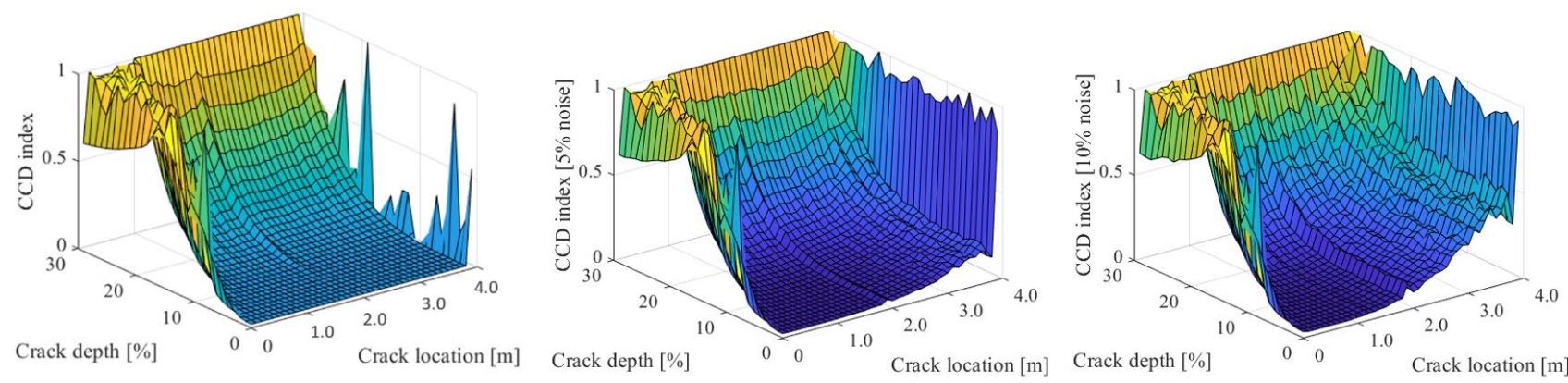

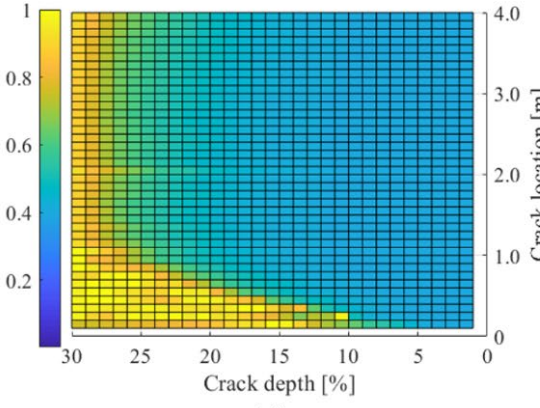

(a)

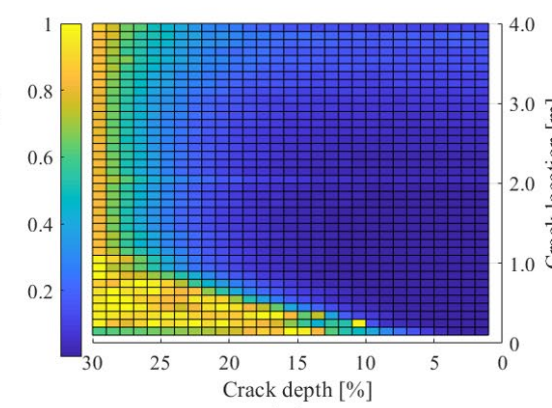

(b)

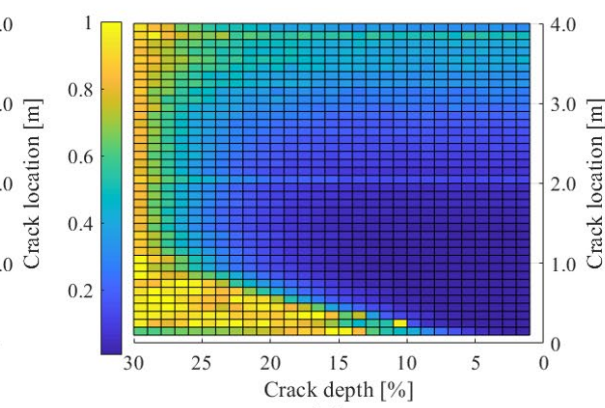

(c)

Figure 16: Two and three-dimension CCD index map without noise (a), $5 \%$ of noise (b), and $10 \%$ of noise (c).

The CCD index displayed in Figure $16(\mathrm{a}-\mathrm{c})$ had a similar behaviour to the RMSD and proved to be a reliable DI, as the RMSD, even for the signal contaminated by noises. In summary, the tendency of the DI was to increase as the crack grew, which can be explained by the fact that the dissipated energy, and therefore, the difference between the signals, increases with the size of the crack. The RMSD and CCD indices presented consistent behaviour and these techniques able to deal with noise in the signals. They gradually varied the DI value as the crack depth increased and were little 
affected by the location of the crack along the beam. The MAPD index presented irregular performance when noise was added and produced a significant numerical error, too. The COV index indicated very high values when the crack position was further from the applied force and had low sensitivity to the crack depth, which can be problematic in structural monitoring and prognosis. By comparing the efficiency and robustness of the DIs, the RSMD and CCD performed better evaluation than the other DIs.

\section{CONCLUDING REMARKS}

In this article, wave propagation responses of undamaged and damaged Euler-Bernoulli beams were used to determine the damage indices. The tests covered a background spectral element method for healthy, semi-infinite, and cracked beams followed by the crack flexibility calculation based on the Castigliano Theorem. The damage indices RMSD, CCD, MAPD, and COV were presented based on the literature and a DI normalisation attempted to compare the performance of each one. The DIs estimated damage metrics by varying crack positions and depth, distance from the crack to the pulse application, and by adding noise to the signals. The responses generated according to the normalised DIs demonstrated that the RMSD and CCD indices are more reliable than the others in terms of sensitivity to changes in crack location and variation of crack depth. The presence of noise affected the responses of the MAPD index, leading to impractical and unreal information. However, the COV index was considered the least effective because it returned DI levels with error over the estimations, as the crack location and depth variation occurred. In general, the addition of noise affected the behaviour of the DIs. The MAPD index was more affected by the noise with inconsistent DI levels. The CCD and RMSD index showed a gradual increase as the crack grew and maintained the estimation with the crack in different positions. They were accurate and indicated DIs to detect and monitor damage combination with the temporal wave signal.

Authors' Contribuitions Conceptualisation, LS Barreto and MR Machado; Methodology, LS Barreto and MR Machado; Investigation, LS Barreto, MR Machado; Writing - original draft, JC Santos, BB de Moura and MR Machado; Writing review \& editing, JC Santos, BB de Moura and MR Machado; Funding acquisition MR Machado and L Khalij; Supervision, MR Machado and L Khalij.

Editor: Pablo Andrés Muñoz Rojas

\section{References}

Apetre, N. and Ruzzene, M. and Hanagud, S. and Gopalakrishnan, S. (2008), A wave-based damage index for the analysis of the filtered response of damaged beams, Journal of Mechanics of Materials and Structures 3(9), 1605-1623.

Al-Oudatallah, J. and Abboud, F. and Khoury, M. and Ibrahim, H. (2017), Overlapping signal separation method using superresolution technique based on experimental echo shape, Advances in Acoustics and Vibration, Article ID 7132038, 9 pages.

Balageas, D. and Fritzen, C. P. and Güemes, A. (2010), Structural health monitoring, Vol. 90, John Wiley \& Sons.

Deluca, A. and Perfetto, D. and Fenza, A. D. and Petrone, G. and Caputo, F. (2020), Guided wave SHM system for damage detection in complex composite structure, Theoretical and Applied Fracture Mechanics 105, 102408.

Doyle, J. F. (1997), Wave propagation in structures: spectral analysis using fast discrete Fourier transforms. Mechanical engineering, Springer-Verlag New York, Inc., New York.

Farrar, C. R. and Worden, K. (2006), An introduction to structural health monitoring. Phil. Trans. R. Soc. A.365303-315.

Flynn, E. B. and Todd, M. and Croxford, B. D. J. and Wilcox, P. (2012), Enhanced detection through low-order stochastic modeling for guided-wave structural health monitoring, Structural Health Monitoring.

Ghaffarzadeh, H. and Barghian, M. and Mansouria, A. and Sadeghi, M. H. (2016), Study on meshfree hermite radial point interpolation method for flexural wave propagation modeling and damage quantification, Latin American Journal of Solids and Structures 13(14). 
Giurgiutiu, V. and Rogers, C. A. (1998), Recent advancements in the electromechanical (e/m) impedance method for structural health monitoring and nde, in: Smart Structures and Materials 1998: Smart Structures and Integrated Systems, Vol. 3329, International Society for Optics and Photonics, pp. 536-548.

Giurgiutiu, V. (2014), Structural health monitoring with piezoelectric wafer active sensors, Elsevier Inc, Academic Press, 2nd edition.

Gopalakrishnan, S. and Ruzzene, M. and Hanagud, S. (2011), Computational techniques for structural health monitoring, Springer Science \& Business Media.

Ho, Y. and Ewins, D. (2000), Numerical evaluation of the damage index, Structural Health Monitoring 1999, 995-1011.

Krawczuk, M. and Palacz, M. and Ostachowics, W. (2002), The dynamic analysis of a cracked Timoshenko beam by the spectral elemet method, Journal of Sound and Vibration 264, 1139-1153.

Krawczuk, M. (2002), Application of spectral beam finite element with a crack and iterative search technique for damage detection, Finite Elements in Analysis and Design 38 (6), 537-548.

Lee, B. and Staszewski, W. (2003), Modelling of lamb waves for damage detection in metallic structures: part ii. Wave interactions with damage, Smart Materials and Structures 12 (5), 815.

Lee, U. (2009), Spectral Element Method in Structural Dynamics, John Wiley \& Sons.

Machado, M.R. and Adhikari, S. and Dos Santos, J. M. C. (2017), A spectral approach for damage quantification in stochastic dynamic systems. Mechanical Systems and Signal Processing 88, 253-273.

Machado, M.R. and Adhikari, S. and Dos Santos, J. M. C. (2018), Spectral element-based method for a onedimensional damaged structure with distributed random properties, Journal of Brazilian Society of Mechanical Sciences and Engineering 40, 214-226.

Machado, M. R. and Santos, J. M. C. (2015), Reliability analysis of damaged beam spectral element with parameter uncertainties, Shock and Vibration 2015, ID 574846, 12 pages.

Machado, M.R. and Appert, A. and Khalij, L. (2019), Spectral formulated modelling of an electrodynamic shaker,Mechanics Research Communications 97, 70-78.

Dutkiewicz, M. and Machado, M.R. (2019), Spectral Approach in Vibrations of Overhead Transmission Lines, IOP Conference Series: Materials Science and Engineering 471(5), 471052029.

Mitra, M. and Gopalakrishnan, S. (2016), Guided wave based structural health monitoring: A review, Smart Materials and Structures 25 (5).

Monaco, E. and Franco, F. and Lecce, L. (2000), Experimental and numerical activities on damage detection using magnetostrictive actuators and statistical analysis, Journal of Intelligent Material Systems and Structures 11 (7), 567-578.

Narayanan, G. and Beskos, D. (1978), Use of dynamic influence coefficients in forced vibration problems with the aid of fast Fourier transform, Computers \& Structures 9 (2), 145-150.

$\mathrm{Ng}, \mathrm{C}$. and Veidt, M. and Lam, H. (2011), Probabilistic damage characterisation in beams using guided waves, Procedia Engineering 14, 490-497.

Ostachowicz, W. and Kudela, P. and Krawczuk, M. and Zak, A. (2012), Guided waves in structures for shm, J. Wiley\&Sons.

Ostachowicz, W. M. (2008), Damage detection of structures using spectral finite element method, Computers \& Structures 86 , 454-462.

Palacz, M. and Krawczuk, M. (2002), Analysis of longitudinal wave propagation in a cracked rod by the spectral element method, Computers \& structures 80 (24), 1809-1816.

Palacz, M. (2018), Spectral methods for modelling of wave propagation in structures in terms of damage detection: a review, Applied Sciences 8 (7), 1124.

Park, S. and Yun, C. and Roh, Y. (2006), PZT-based active damage detection techniques for steel bridge components, Smart Materials and Structures 15, 957.

Rizzo, P. and Lanza, F. (2006), Feature extraction for defect detection in strands by guided ultrasonic waves, Structural Health Monitoring 5(3). 
Sharma, V. and Ruzzene, M. and Hanagud, S. (2006), Damage index estimation in beams and plates using laser vibrometry 44 (4).

Sohn, H. and Farrar, C. R. and Hemez, F. M. and Shunk, D. D. and Stinemates, D. W. and Nadler, B. R. and Czarnecki, J. J. (2003), A review of structural health monitoring literature: 1996-2001, Los Alamos National Laboratory, USA.

Stawiarski, A. and Muc, A. (2019), On transducers localisation in damage detection by wave propagation method, Sensors MDPI 19, 1-18.

Su, Z. and Ye, L. (2009), Identication of damage using Lamb waves: from fundamentals to applications, Vol. 48, Springer Science \& Business Media.

Tada, H. and Paris, P. C. and Irwin, G. R. (1973), The stress analysis of cracks, Handbook, Del Research Corporation.

Tseng, K. K. and Naidu, A. (2002), Non-parametric damage detection and characterization using smart piezoceramic material, Smart Materials and Structures 11 (3) 371.

Tung, Y.-K. and Yen, B. C. (2005), Hydrosystems engineering uncertainty analysis, McGraw- Hill.

Zhang, H. and Shen, W. and Li, D. and Zhang, X. and Chen, B. (2018), Non-destructive ultrasonic testing in rod structure with a novel numerical Laplace based wavelet finite element method, Latin American Journal of Solids and Structures 15(7), 2606262. 